Original article

\title{
EFFECTS OF POST-HATCH NUTRITION OF ARGININE, LYSINE, METHIONINE AND THREONINE ON PERFORMANCE AND INTESTINAL MORPHOLOGY IN BROILER CHICKENS
}

\author{
A. SADRZADEH ${ }^{1}$, H. HASSANPOUR ${ }^{2}$, M. H. MEHRAEI HAMZE- \\ KOLAEI $^{3}$, P. FARHADNIYA ${ }^{1}$, M. R. EHSAN ${ }^{4} \&$ S. S. TOHIDI FAR ${ }^{3}$ \\ ${ }^{1}$ Department of Clinical Sciences, Faculty of Veterinary Medicine, Islamic Azad University, \\ Garmsar Branch, Garmsar, Iran; ${ }^{2}$ Department of Basic Sciences (Physiology division), \\ Faculty of Veterinary Medicine, Shahrekord University, Shahrekord, Iran; ${ }^{3}$ Department of \\ Clinical Sciences, Faculty of Veterinary Medicine, Shahrekord University, Shahrekord, \\ Iran; ${ }^{4}$ Department of Clinical Science, Faculty of Veterinary Medicine,
}

Tehran University, Tehran, Iran

\section{Summary}

Sadrzadeh, A., H. Hassanpour, M. H. Mehraei Hamzekolaei, P. Farhadniya, M. R. Ehsan \& S. S. Tohidi Far, 2015. Effects of post-hatch nutrition of arginine, lysine, methionine and threonine on performance and intestinal morphology in broiler chickens. Bulg. J. Vet. Med., 18, No. 4, 294-303.

To investigate the effects of post-hatch nutrition of arginine, lysine, methionine and threonine on performance and intestinal morphology of broilers, 180 day-old Ross-308 were assigned to 6 treatments. Chickens were provided post-hatch ( $48 \mathrm{~h}$ ) feeding of each amino-acid in 4 groups. Chickens in other 2 treated groups received no feed and water (fasting group) or just water (watering group). On day 14 , performance parameters were determined, and then all chickens from each treatment were killed. After weighing body organs, the length of segments of small intestine were measured and prepared for evaluation of intestinal morphology. The mean body weight and FCR between watering, fasting, methionine and threonine groups did not differ while they all were greater than lysine fedchickens $(\mathrm{P}<0.05)$. In comparison with other groups, the lamina propria thickness of duodenum and ileum were greater in methionine and threonine groups, and in jejunum it was more in methionine group $(\mathrm{P}<0.05)$. The sum of measured villus surface area was higher and lower in threonine and lysine groups respectively $(\mathrm{P}<0.05)$. In conclusion, among the 4 amino acids, post-hatch feeding of threonine has beneficial effects especially on the growth of intestine. In contrast, lysine has adverse effects on the body performance and intestinal morphology.

Key words: amino acid, broiler, intestine, post-hatch feeding 


\section{INTRODUCTION}

Several studies have determined the critical role of post-hatch nutrition of fast growing broiler chicks on their performance (Madsen et al., 2004; Noy \& Uni, 2010; Mahmoudi et al., 2012). Early nutrition influences development and growth of chickens over several days (Bigot et al., 2003; Quentin et al., 2005). Industrial chicks are usually hatched for two days; then they are sexed, vaccinated, and finally transported to industrial farms. During this time (2-3 days of age), newly hatched chicks do not feed or drink anything. Madsen et al. (2004) showed that this condition could delay growth of chicks. Pinchasov (1991) and Noy \& Sklan (1999) had also confirmed that inaccessibility of chicks to feed and water in the first days of life, decreased body weight and other body performance. In contrast, shortening the time of deprivation in post hatched chicks, improved body growth and developed intestine, pancreas and immune system (Jin et al., 1998; Madsen et al., 2004). It has been suggested that an accumulation of nutrients in crop of chicks may contribute in the development of digestive system even before arriving to industrial farm (Bhanja et al., 2010).

Several experiments have shown that amino acids in chickens are an important requirement for development of different organs of body, and the relation between amino acid/protein requirements and factors such as sex, age, heat stress, energy concentration (Garcia et al., 2006; Bastianelli et al., 2007; Fischer da Silva et al., 2007). Pesti \& Miller (1997) identified that amino acids in optimal levels were not suitable for the maximisation of body performance e.g., weight gain, feed conversion rate and growing of breast muscle. Recent studies determined the positive effects of many essential amino acids on body weight after feeding to post-hatched chickens (Noy \& Sklan, 2002; Stringhini et al., 2009). The present research was aimed at evaluating the effects of the first amino acids (lysine, arginine, threonine and methionine) eaten post hatching (from 0 to $48 \mathrm{~h}$ ) on subsequent (14 days of age) body performance and intestinal morphology. This study investigated whether the intake of a single amino acid in the early life could have positive effects on the mentioned parameters during the further growth period until day 14 .

\section{MATERIALS AND METHODS}

\section{Animals, management and treatments}

One hundred and eighty broiler chicks of either sex from Ross 308 breed were used in this study. Each bird was weighed in the hatchery and then transported immediately to the neighbour research farm without any loss of time (less than $1 \mathrm{~h}$ ). The chicks were housed in pens $1 \times 1 \mathrm{~m}$ of size and wood shavings flooring. They were randomly distributed to 6 treatments with 3 equal replicates of 10 chicks per pen. Equal numbes of both sexes were settled in each treatment groups. The treated groups were assigned as: 1) No feed and water in $48 \mathrm{~h}$ of post-hatch (fasting group); 2) No feed but free access to water in $48 \mathrm{~h}$ of post-hatch (watering group); 3 ) Intubation of arginine (120\% of NRC; Kidd et al., 2001) in $48 \mathrm{~h}$ of post-hatch (arginine group); 4) Intubation of lysine (110\% of NRC; Kidd et al., 1997) in $48 \mathrm{~h}$ of post-hatch (lysine group); 5) Intubation of methionine $(130 \%$ of NRC; Ahmed \& Abbas, 2011) in $48 \mathrm{~h}$ of post-hatch ( methionine group); and 6) Intubation of threonine (100\% of NRC; Kidd et al., 1997) in $48 \mathrm{~h}$ of post-hatch (threonine group). 
Effects of post-hatch nutrition of arginine, lysine, methionine and threonine on performance ....

Handling and intubation were done in a humane way according to Iranian animal welfare regulations. After $48 \mathrm{~h}$ of rearing, all treated groups were fed until 14 days of age a starter diet based on corn/soybean (13 MJ metabolisable energy $/ \mathrm{kg}$ of diet, $230 \mathrm{~g} / \mathrm{kg}$ crude protein) formulated to meet requirements for broilers according to NRC (1994).

\section{Performance parameters and carcass traits}

Body weights of broilers were recorded post-hatch and after $14^{\text {th }}$ day of age. Feed intake and weight gain were recorded at the end of the $14^{\text {th }}$ day. The ratio between feed intake and weight gain was calculated to offer the feed conversion efficiency (FCR).

At the end of the experiment (14 days of age), all chickens were weighed and killed by decapitation, then the internal organs (i.e., bursa of Fabricius, liver, gizzard, proventriculus, spleen and duodenum+pancreas) were weighed and the per cents of organ weight/body weight (BW) were calculated. Moreover, the length of intestine (duodenum - from gizzard's end to the end of the pancreas; jejunum from the end of the pancreas to the vitelline diverticulum; ileum - from the vitelline diverticulum to the junction of caeca; and rectum - from the junction of caeca to cloaca) were measured in millimeters using a ruler.

\section{Morphometric analysis of the intestine}

The intestinal morphometric variables i.e., villus sizes (length, width, surface area and lamina propria thickness) were evaluated in the duodenum, jejunum and ileum of killed birds at 14 days of age according to Sakamoto et al. (2000). Briefly, after fixation of the mentioned segments of intestine in Clark solution, each segment was divided into 3 sections along its length. Sections were stained by periodic acid Schiff (PAS) reagent, then rows of villi were separated in thickness of the sections, transferred over glass slides and covered with a cover-slip. Eyepiece graticules $(10 \times)$ and magnification of $\times 100$ were used in microscope to measure villus sizes in these samples. The villus length and width were used to calculate villus surface area according to formula $\pi \times($ villus width $) \times($ villus length $)$, as described by Sakamoto et al. (2000). The lamina propria thickness was measured at the space between base of the villus and top of the muscularis mucosa. This experiment was done for 30 chickens/treatment.

\section{Statistical analysis}

All data are offered as mean \pm SE. Intestinal and performance parameters were compared between chicken groups using one-way ANOVA with Tukey's post hoc test (SigmaStat 3.1. Ink). The $\mathrm{P}$ values less than 0.05 were statistically significant.

\section{RESULTS}

\section{Growth performance and carcass traits}

The mean initial body weight was the same between different groups of chickens (data not shown). At the end of the experiment (day 14), the mean body weight of chickens between watering, fasting, methionine and threonine groups did not differ while they all were greater than lysine group $(\mathrm{P}<0.05)$. The feed conversion ratio was higher in lysine group than in other groups $(\mathrm{P}<0.05)$. The per cent of bur$\mathrm{sa} / \mathrm{BW}$ in the fasting group was higher than in arginine, lysine, methionine and threonine groups $(\mathrm{P}<0.05)$. This parameter in lysine group was lower than in all 
A. Sadrzadeh, H. Hassanpour, M. H. Mehraei Hamzekolaei, P. Farhadniya, M. R. Ehsan ...
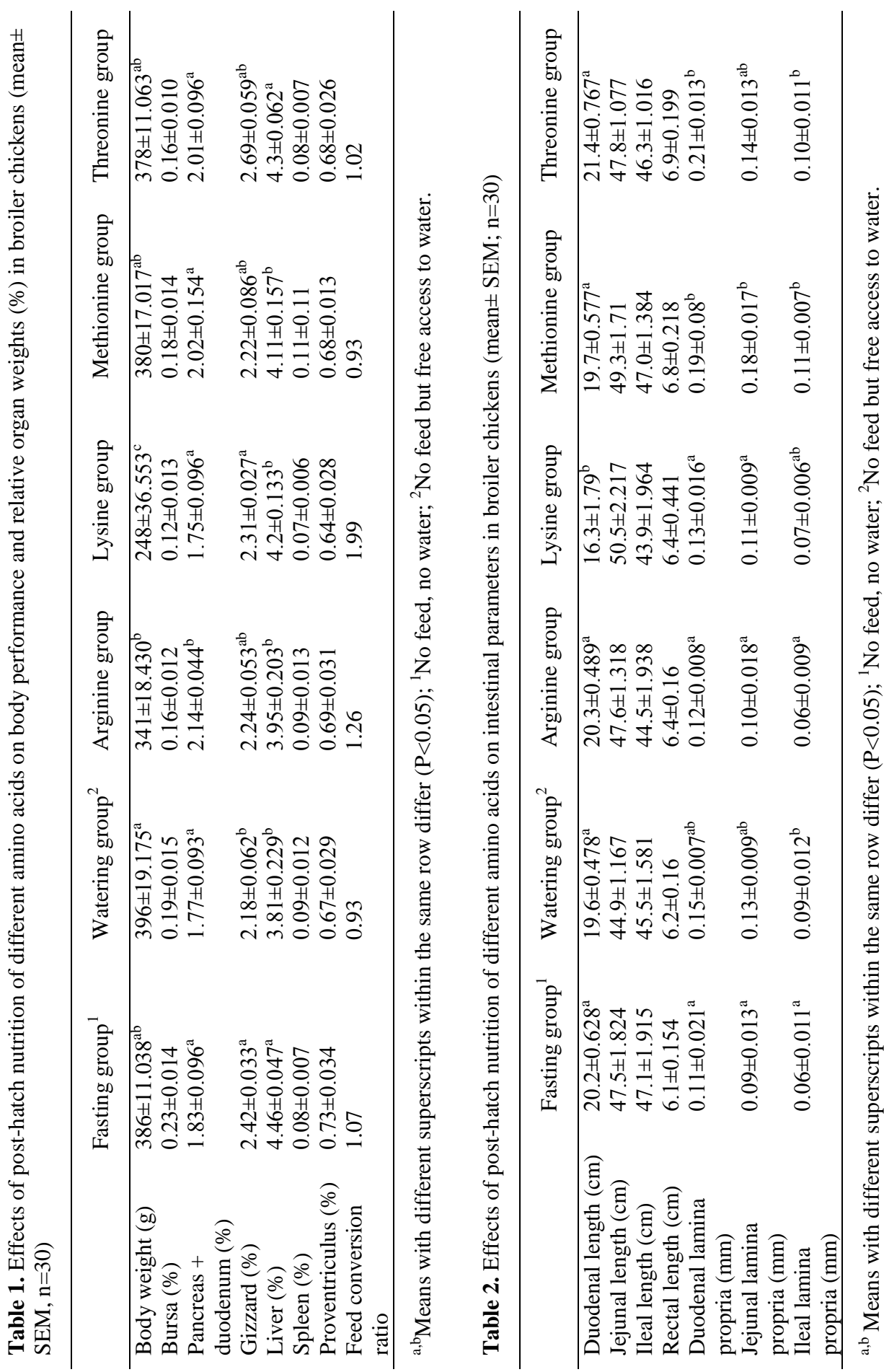

BJVM, 18, No 4 
Effects of post-hatch nutrition of arginine, lysine, methionine and threonine on performance ....

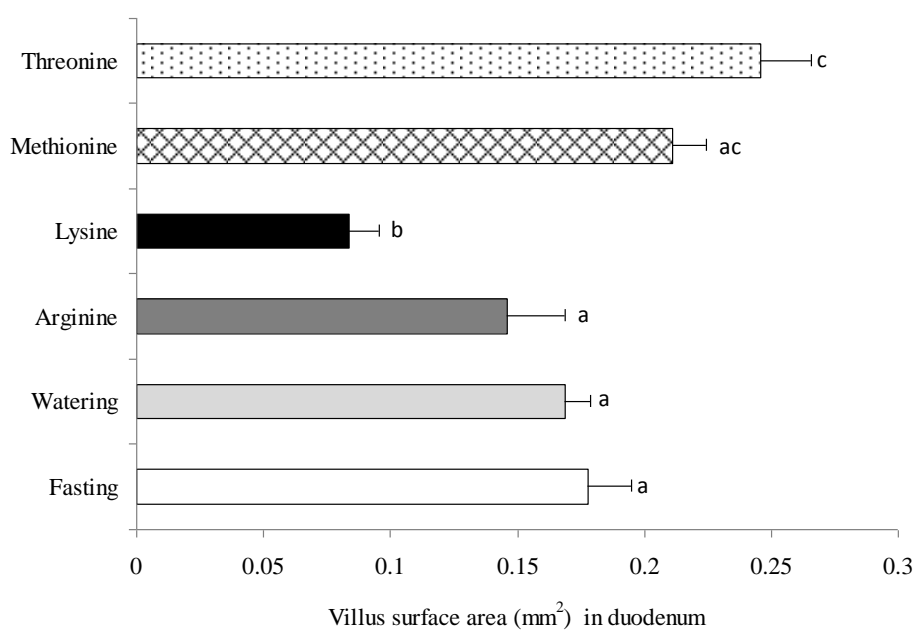

Fig. 1. The villus surface area in duodenum of broilers at 14 days of age, post-hatch fed with arginine, lysine, methionine and threonine, deprived from water and food (fasting) or with only access to water (watering). All data are presented as mean \pm SEM $(n=30)$. Means with different indices differ significantly $(\mathrm{P}<0.05)$.

other groups $(\mathrm{P}<0.05)$. The percent of pancreas+duodenum/BW, spleen/BW and proventriculus/BW in lysine group were lower than other groups of chickens. The per cent of liver/ BW and gizzard/BW were statistically different among experimental groups (Table 1).

\section{Intestinal morphometric assessment}

In lysine group, the duodenum were shorter than in other groups of chickens $(\mathrm{P}<0.05)$ while the length of jejunum, ileum and rectum did not differ among experimental groups. The lamina propria thickness of duodenum in methionine and threonine groups were greater than fasting, arginine and lysine groups $(\mathrm{P}<0.05)$. The lamina propria thickness of jejunum in methionine group was also greater than in fasting, arginine and lysine groups $(\mathrm{P}<0.05)$. The ileal lamina propria thickness was higher in methionine and threonine groups than in fasting and arginine groups $(\mathrm{P}<0.05)$ (Table 2).
The villus surface area was lower in the duodenum of lysine group at day 14 compared to other experimental groups of chickens $(\mathrm{P}<0.05)$, whereas this parameter in threonine group was higher than in other groups $(\mathrm{P}<0.05$; Fig. 1$)$. The villus surface area was increased in the jejunum of threonine group compared to other groups of chickens $(\mathrm{P}<0.05)$, although significant variations between other groups were also observed (Fig. 2). The villus surface area in the ileum of fasting and lysine groups was lower than in other groups $(\mathrm{P}<0.05)$; the villus surface area of ileum among the other groups was not significant, although this parameter in threonine group was higher (Fig. 3).

As presented on Fig. 4, the sum of measured villus surface area in three intestinal parts at the end of experiment was higher and lower in threonine and lysine groups respectively than in other groups of chickens $(\mathrm{P}<0.05)$. 


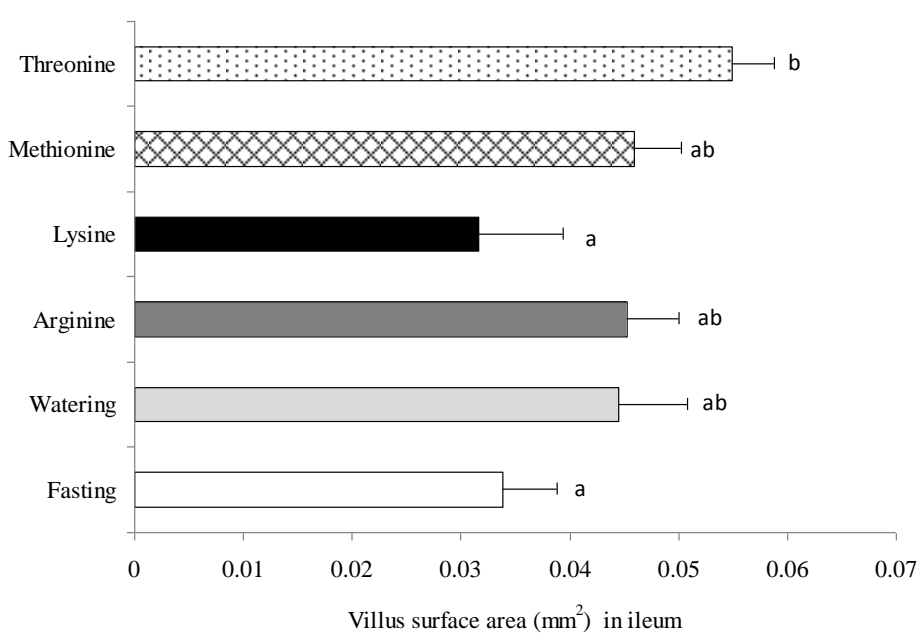

Fig. 2. The villus surface area in ileum of broilers at 14 days of age, post-hatch fed with arginine, lysine, methionine and threonine, deprived from water and food (fasting) or with only access to water (watering). All data are presented as mean $\pm \operatorname{SEM}(n=30)$. Means with different indices differ significantly $(\mathrm{P}<0.05)$.

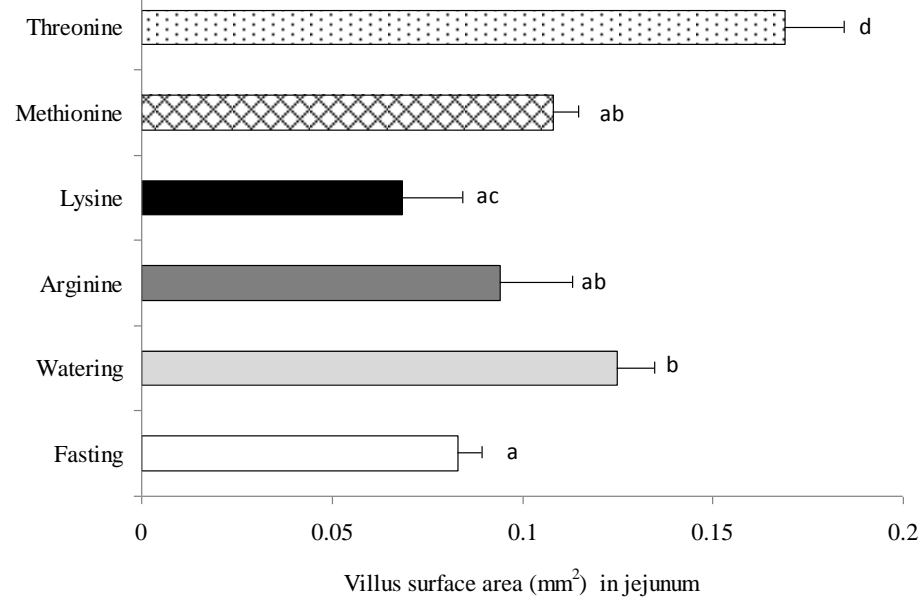

Fig. 3. The villus surface area in jejunum of broilers at 14 days of age, post-hatch fed with arginine, lysine, methionine and threonine, deprived from water and food (fasting) or with only access to water (watering). All data are presented as mean \pm SEM $(n=30)$. Means with different indices differ significantly $(\mathrm{P}<0.05)$.

\section{DISCUSSION}

Many studies examined the effects of post-hatch nutrition on chickens, which indicated that feeding in this period of rearing, could imply positive effects on chicken performance in comparison with feed deprivation (Gonzales et al., 2003; Madsen et al., 2004; Bhanja et al., 2010; Noy \& Uni, 2010; Mahmoudi et al., 2012). Earlier, it seemed that nutrients of 
Effects of post-hatch nutrition of arginine, lysine, methionine and threonine on performance ....

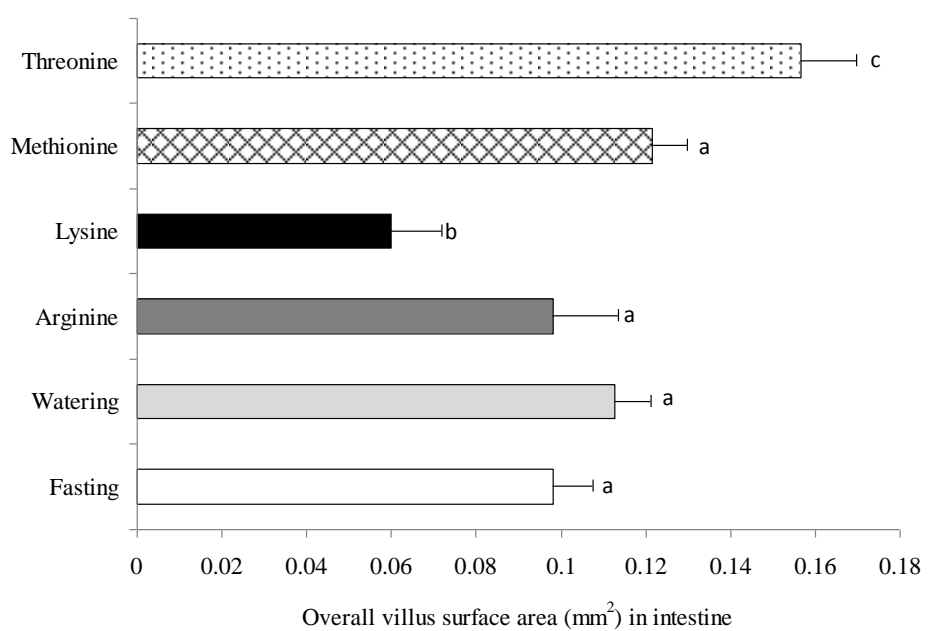

Fig. 4. Overall villus surface area in intestine of broilers at 14 days of age, post-hatch fed with arginine, lysine, methionine and threonine, deprived from water and food (fasting) or with only access to water (watering). All data are presented as mean \pm SEM $(n=30)$. Means with different indices differ significantly $(\mathrm{P}<0.05)$.

yolk including proteins and carbohydrates were enough for growth and developments of chickens during post-hatch period. Next studies cleared that early feeding of chickens with different percent of crude protein, starch, soybean oil or casein could enhance growth of different apparatus of body such as digestive and immune systems (Madsen et al., 2004; Pinheiro et al., 2004; Bhanja et al., 2010; Yadav et al., 2011).

In the present study, the broiler chickens were fed 4 amino acids for $48 \mathrm{~h}$, and their doses were suggested by previous studies. In addition, two groups of chicken received no feed or just water for $48 \mathrm{~h}$. After 48 h, all chickens were fed a standard diet. At the end of experiments (day 14), comparison of body weight and FCR between experimental groups showed that feeding of chickens with amino acids not only did not have any advantages in those aspects but also feeding with lysine had negative effects. Stringhini et al. (2009) also reported that supplement of methio- nine to the chickens during pre-starter period did not influence weight gain, feed intake and feed to gain ratio; although, a complex of methionine, lysine, threonine and tryptophan improved feed to gain ratio. In the present study, many internal organs of body were weighed, in which results were different among experimental groups; but it could be deduced that growth of organs such as bursa, spleen, proventriculus, pancreas and intestine (duodenal length) in lysine group was slower than those in other chickens. The reason for this result is not clear. Perhaps, the used dose of lysine in this study, interacted with other yolk amino acids. Chamruspollert et al. (2002) found an interrelationship among lysine, methionine and arginine. They suggested that excess amount of lysine could increase arginase activity, which reduced the amount of available arginine. Excess dietary lysine also decreased transamidinase activity leading to diminished creatine biosynthesis in the muscles. On the other 
hand, they reported that an increase in arginine caused growth and feed intake depression while increasing methionine in the diet improved body performance.

Despite of our data on the body performance and carcass traits, the effect of the utilized amino acids especially methionine and threonine on the intestinal morphology was noticeable. The thickness of lamina propria in 3 parts of intestine was increased when feeding these two amino acids, which was apparently due to more development of Lieberkühn's glands in the space of lamina propria. This result may influence intestinal secretion in chickens. Nir et al. (1996), Pinheiro et al. (2004) and Noy \& Uni (2010) reported that development of digestive tract plays an individual role in the early growth of chickens because nutrients in post-hatch stimulated the production of digestive enzymes. Uni et al. (1998) examined the effects of early withholding feed on morphometric parameters of chick intestine. They observed a considerable decrease of villus volume in the jejunum and an irregular morphology in jejunal crypts. Scanning electron microscopy also confirmed some alterations (included clumping of microvilli and abnormal crypt structure) in the morphology of intestine in the mentioned chickens. Bigot et al. (2003) and Fischer da Silva et al. (2007) also reported that early deprivation delayed the growth of intestine while amino acids such as glutamine increased intestinal surface area and improved growth of intestinal villi. These data are in agreement with our results, which demonstrated that villus surface area as index of villus sizes in the intestine was increased. This increase indicates better growth of intestinal villi. Apparently, the positive effect of threonine in this aspect was predominant, which has been shown well in calculating the overall vil- lus surface area. On the other hand, lysine had no effect or adverse effect on villus surface area. However, many studies confirmed that post-hatch nutrition improved the intestinal parameters (Bigot et al., 2003; Tabeidian et al., 2011; Yadav et al., 2011) but as mentioned above, the interactions between amino acids could increase body requirements to many of them. This situation between lysine, methionine and arginine has been confirmed (Chamruspollert et al., 2002) which is probably the reason of adverse effects on the intestinal development. It must be noticed that in the hatching chick, the small intestine matures largely in the immediate post-hatch days, which occurs with intensive cryptogenesis, enterocyte maturation to functional forms (Geyra et al., 2001). On the other hand, intestinal development needs amino acids (Foye et al., 2007; Wijtten et al., 2010). Then, any malnutrition with amino acids may influence the intestinal development at posthatch period, which would be background for diminished growth of intestine in the rearing period.

It is concluded that among the 4 amino acids (arginine, lysine, methionine and threonine), post-hatch feeding of threonine has beneficial effects especially on the growth of intestine, although its effects on the broiler chicken performance were not considerable. In contrast, lysine had adverse effects on the body performance and intestinal morphology.

\section{ACKNOWLEDGMENTS}

This work was supported by the funds awarded by the Vice Chancellor for Research of Islamic Azad University. 
Effects of post-hatch nutrition of arginine, lysine, methionine and threonine on performance ....

\section{REFERENCES}

Ahmed, M. E. \& T. E. Abbas, 2011. Effects of dietary levels of methionine on broiler performance and carcass characteristics. International Journal of Poultry Science, 10, 147-151.

Bastianelli, D., M. Quentin, I. Bouvarel, C. Relandeau, P. Lescoat, M. Picard \& S. Tesseraud, 2007. Early lysine deficiency in young broiler chicks. Animal Science, 1, 587-594.

Bhanja, S. K., C. Anjali Devi, A. K. Panda \& G. Shyam Sunder, 2010. Effect of posthatch nutrient intubation on performance, intestinal growth, meat yield and immune response in broiler chickens. Asian-Australasian Journal of Animal Sciences, 23, 515-520.

Bigot, K., S. Mignon-Grasteau, M. Picard \& S. Tesseraud, 2003. Effects of delayed feed intake on body, intestine, and muscle development in neonate broilers. International Journal of Poultry Science, 82, 781-788.

Chamruspollert, M., G. M. Pesti \& R. I. Bakalli, 2002. Dietary interrelationships among arginine, methionine, and lysine in young broiler chicks. British Journal of Nutrition, 88, 655-660.

Fischer da Silva, A. V., A. Maiorka, S. A. Borges, E. Santin, I. C. Boleli \& M. Macari, 2007. Surface area of the tip of the enterocytes in small intestine mucosa of broilers submitted to early feed restriction and supplemented with glutamine. International Journal of Poultry Science, 6, 31-35.

Foye, O. T., P. R. Ferket \& Z. Uni, 2007. The effects of in ovo feeding arginine, $\beta$ hydroxy- $\beta$-methyl-butyrate, and protein on jejunal digestive and absorptive activity in embryonic and neonatal turkey poults. International Journal of Poultry Science, 86, 2343-2349.

Garcia, A. R., A. B. Batal \& D. H. Baker, 2006. Variations in the digestible lysine requirement of broiler chickens due to sex, performance parameters, rearing environ- ment and processing yield characteristics. International Journal of Poultry Science, 85, 498-504.

Geyra, A., Z. Uni \& D. Sklan, 2001. Enterocyte dynamics and mucosal development in the posthatch chick. International Journal of Poultry Science, 80, 776-782.

Gonzales, E., N. Kondo, E. S. Saldanha, M. M. Loddy, C. Careghi \& E. Decuypere, 2003. Performance and physiological parameters of broiler chickens subjected to fasting on the neonatal period. International Journal of Poultry Science, 82, 12501256.

Jin, S. H., A. Corless \& L. J. Sell, 1998. Digestive system development in posthatch poultry. World's Poultry Science Journal, 54, 335-345.

Kidd, M. T., B. J. Kerr \& N. B. Anthony, 1997. Dietary interactions between lysine and threonine in broilers. International Journal of Poultry Science, 76, 608-614.

Kidd, M. T., E. D. Peebles, S. K. Whitmarsh, J. B. Yeatman \& R. F. Wideman, 2001. Growth and immunity of broiler chicks as affected by dietary arginine. International Journal of Poultry Science, 80, 15351542.

Madsen, J. H. R., G. Su \& P. Sorensen, 2004. Influence of early or late start of first feeding on growth and immune phenotype of broilers. British Poultry Science, 45, 210-222.

Mahmoudi, S., A. Mirza-Aghazadeh, N. M. Sis, K. Hatefinezhad \& A. Gorbani, 2012. Effects of delayed post hatch feed intake on performance of broilers kept in cages. European Journal of Experimental Biology, 2, 843-845.

Nir, I., Z. E. A. Nistan, P. Dunnington \& P. B. Siegel, 1996. Aspects of food intake restriction in domestic fowl: Metabolic and genetic considerations. World's Poultry Science Journal, 52, 251-266.

Noy, Y. \& D. Sklan, 1999. Energy utilization in newly hatched chicks. International Journal of Poultry Science, 78, 1750-1756. 
Noy, Y. \& D. Sklan, 2002. Nutrient use in chicks during the first week posthatch. International Journal of Poultry Science, 81, 391-399.

Noy, Y. \& Z. Uni, 2010. Early nutritional strategies. World's Poultry Science Journal, 66, 639.

NRC, 1994. Nutrient Requirements of Poultry. $9^{\text {th }}$ rev. edn, National Academic Press, Washington, DC.

Pesti, G. M. \& B. R. Miller, 1997. Modelling for precision nutrition. Journal of Applied Poultry Research, 6, 483-494.

Pinchosav, Y., 1991. Relationship between the weight of hatching eggs and subsequent early performance of broiler chicks. British Poultry Science, 32, 109-115.

Pinheiro, D. F., V. C. Cruz \& M. L. V. Paulino, 2004. Effect of early feed restriction and enzyme supplementation on digestive enzyme activities in broilers. International Journal of Poultry Science, 83, 1544-1550.

Quentin, M., I. Bouvarel \& M. Picard, 2005. Effects of the starter diet, light intensity, and essential amino acids level on growth and carcass composition of broilers. Journal of Applied Poultry Research, 14, 6976.

Sakamoto, K., H. Hirose, A. Onizuka, M. Hayashi, N. Futamura, Y. Kawamura \& T. Ezaki, 2000. Quantitative study of changes in intestinal morphology and mucus gel on total parenteral nutrition in rats. Journal of Surgical Research, 94, 99-106.

Stringhini, J. H., M. L. Andrade, S. A. G. Xavier, M. A. Andrade, C. S. Minafra \& M. B. Café, 2009. Protein and amino acid supplementation levels for broilers in prestarter ration. International Journal of Poultry Science, 8, 946-951.
Tabeidian, S. A., A. Samie, J. Pourreza \& G. Sadeghi, 2011. Effect of fasting or posthatch diet's type on Intestinal morphology in broilers. In: Proceedings of International Conference on Life Science and Technology (ICLST 2011).

Uni, Z., S. Ganot \& D. Sklan, 1998. Posthatch development of mucosal function in the broiler small intestine. International Journal of Poultry Science, 77, 75-82.

Wijtten, P. J. A., E. Hangoor, J. Sparla \& M. W. A. Verstegen, 2010. Dietary amino acid levels and feed restriction affect small intestinal development, mortality, and weight gain of male broilers. International Journal of Poultry Science, 89, 14241439.

Yadav, G. B., A. S. Kadam, A. M. Pachpande, S. B. Lambate, V. D. Lonkar, S. Maini \& K. Ravikanth, 2011. Post hatch histomorphological studies of small intestinal development in chicks fed with herbal early chick nutritional supplement. International Journal of Poultry Science, 9 , 851-855.

Paper received 11.10.2014; accepted for publication 22.01.2015

\section{Correspondence:}

Mohammad Hasan Mehraei Hamzekolaei Department of Clinical Sciences, Faculty of Veterinary Medicine, Shahrekord University, Shahrekord, Iran e-mail: mehraei.mohammad@stu.sku.ac.ir 